\title{
Inhibition of Activin/Myostatin signalling induces skeletal muscle hypertrophy but impairs mouse testicular development
}

\author{
Danielle Vaughan (1), Olli Ritvos (2), Robert Mitchell (1), Oliver Kretz (3), Maciej \\ Lalowski (4), Helge Amthor (5), David Chambers (6), Antonios Matsakas (7), Arja \\ Pasternack (2), Henry Collins-Hooper (1), Randy Ballesteros (8), Tobias B. Huber (3), \\ Bernd Denecke (9), Darius Widera (10), Abir Mukherjee (8), Ketan Patel (1)
}

(1) School of Biological Sciences, University of Reading, UK; (2) Department of Bacteriology and Immunology, University of Helsinki, Helsinki, Finland; (3) III Department of Medicine, University Medical Center Hamburg-Eppendorf, Hamburg, Germany; (4) Department of Biochemistry and Developmental Biology, HiLIFE, Meilahti Clinical Proteomics Core Facility, University of Helsinki, Helsinki, Finland; (5) Versailles Saint-Quentin-en-Yvelines University, INSERM U1179, LIA BAHN CSM, Montigny-le-Bretonneux 78180, France; (6) Functional Genomics, King's college, London, England; (7) Molecular Physiology Laboratory, Centre for Atherothrombosis \& Metabolic Disease, Hull York Medical School, Hull, UK; (8) Royal Veterinary College, London, UK; (9) RWRTH Aachen University, Aachen, Germany; (10) School of Pharmacy, University of Reading, UK

This article is distributed under the terms of the Creative Commons Attribution Noncommercial License (CC BY-NC 4.0) which permits any noncommercial use, distribution, and reproduction in any medium, provided the original author(s) and source are credited.

\begin{abstract}
Numerous approaches are being developed to promote post-natal muscle growth based on attenuating Myostatin/Activin signalling for clinical uses such as the treatment neuromuscular diseases, cancer cachexia and sarcopenia. However there have been concerns about the effects of inhibiting Activin on tissues other than skeletal muscle. We intraperitoneally injected mice with the Activin ligand trap, sActRIIB, in young, adult and a progeric mouse model. Treatment at any stage in the life of the mouse rapidly increased muscle mass. However at all stages of life the treatment decreased the weights of the testis. Not only were the testis smaller, but they contained fewer sperm compared to untreated mice. We found that the hypertrophic muscle phenotype was lost after the cessation of sActRIIB treatment but abnormal testis phenotype persisted. In summary, attenuation of Myostatin/Activin signalling inhibited testis development. Future use of molecules based on a similar mode of action to promote muscle growth should be carefully profiled for adverse side-effects on the testis. However the effectiveness of sActRIIB as a modulator of Activin function provides a possible therapeutic strategy to alleviate testicular seminoma development.
\end{abstract}

Key Words: Muscle hypertrophy, Activin, Myostatin, adverse effects, neuromuscular diseases, Mossman-Pacey paradox, testis.

Eur J Transl Myol 30 (1): xx1-x17, 2020

Skeletal muscle is an adaptable tissue capable of changing its mass and composition to meet the physiological needs of the human body. ${ }^{1}$ The amount of protein present in any organ is controlled by the rate of its synthesis and breakdown and it has been estimated that, in humans, it has a turnover rate of $5.7 \mathrm{~g} / \mathrm{kg} / \mathrm{day},{ }^{2}$ with skeletal muscle proteins having an average half-life of only 20 days $^{3}$ Therefore, the regulation of skeletal muscle protein content is highly dynamic, and any failures in the mechanisms responsible may manifest in muscle loss or atrophy. A variety of clinically relevant factors have been described that lead to muscle loss including genetic mutations (e.g. Duchenne Muscular Dystrophy-DMD), diseases in non-skeletal muscles that produce muscle wasting molecules (as in cancer cachexia) and age-related muscle wasting (sarcopenia). Given that muscle wasting is such a common feature of human life, it is hardly surprising that great efforts have been made to understand muscle loss with a view of attenuating or reversing this process. Many different molecules have received interest from a therapeutic perspective over the last few decades including anabolic 
steroids, ${ }^{4}$ growth hormones, ${ }^{5}$ and IGF- $1 .{ }^{6}$ However all of these are associated with adverse side effects.

The landscape of muscle growth promoting molecules was revolutionised following the discovery of Myostatin. ${ }^{7}$ In a ground-breaking study, the group of SeJin Lee showed that this member of the TGF- $\beta$ family of secreted signalling proteins was predominantly expressed by skeletal muscle during embryogenesis and its deletion in mouse (the $\mathrm{Msth}^{-/-}$line) led to a huge increase in muscle mass as a consequence of muscle hyperplasia and fibre hypertrophy. ${ }^{7}$ Thereafter mutations in the Myostatin (Mstn) gene have been found in a number of mammalian species including one case in humans all of which manifest with muscle hypertrophy. ${ }^{8}$ Thereafter a number of strategies have been developed to attenuate the activity of Myostatin during post-natal life in-order to promote muscle growth for therapeutic uses. These include: antibodies and a variety of proteins (including Follistatin, ${ }^{9}$ GASP $-1,{ }^{10}$ LTBP- $-{ }^{11}$ ) that bind to Myostatin and prevent it from functioning normally, and propeptide regions that re-associate with Myostatin. ${ }^{12}$ Alternative anti-Myostatin strategies have been developed based on ligand-receptor interactions. Myostatin and related TGF $\beta$ ligands such as Activin exert their action by binding to a heterotetrameric receptor complex comprised of two Type I and two Type II receptors on their target cells. Myostatin/Activin signalling is mediated by either ALK4 or ALK7 and ACVR2A or ACVR2B (henceforth ActRIIA or ActRIIB, respectively). ${ }^{13}$ This knowledge has been exploited to develop ligand blocking antibodies to ActRIIB which have been shown to promote robust muscle hypertrophy. ${ }^{14}$ In addition, we and others developed a stabilised peptide containing the ligand binding domain of ActRIIB (hereafter referred to as SActRIIB) which also promotes skeletal muscle growth. ${ }^{15}$ The latter two strategies are particularly attractive in terms of muscle wasting therapies since it has been shown that Activin as well as Myostatin signal through the ActRIIB receptor to inhibit muscle growth; sActRIIB promotes muscle growth in the $\mathrm{Mstn}^{-/}$mice.16 However studies have shown the sActRIIB binds a number of forms of Activin as well as GDF11 and BMPs 2,7, 9 and 10. ${ }^{17-20}$

The broad ligand-binding spectrum of sActRIIB raises the concern that although it is able to promote muscle growth, it may interfere with other cellular processes. Indeed, the use of a sActRIIB molecule in primates was shown to increase pancreas and spleen weights as well as interfering with glucose homeostasis. ${ }^{21}$

In this study we examined the impact of sActRIIB on the development of the testis and sperm as both processes have been shown to be regulated by Activin. The mammalian testis is a complex organ composed of several cell types, organised in structurally distinct domains that undertake its two main functions. The seminiferous tubule is where spermatogenesis occurs and between these convoluted tubules are the interstitial cells, blood vessels and the sites of male hormone production.
Within the seminiferous tubule, surrounded by a basement membrane are Sertoli cells (SC). These are somatic cells that interact with the spermatogenic cells and act as support cells for spermatogenesis. SC proliferate and differentiate in the postnatal testis and the maximum number of SC per testis is established by day 15 in mice. Spermatogenesis is closely linked to SC function. At the basal surface of the seminiferous tubule between the SCs reside spermatogonial stem cells which gives rise to spermatogonia. Spermatogonia undergo a series of mitotic divisions that lead to spermatogonial renewal and differentiation into Type A and then Type B spermatogonia. These then undergo mitosis and differentiation to produce primary or preleptotene spermatocytes. Importantly, these diploid cells traverse through the blood-testis barrier (BTB), a tight junction between two adjacent SCs to reach the adluminal compartment. The BTB physically prevent the movement of molecules between the circulation and the adluminal compartment, isolating the adluminal compartment and sperm from the rest of the periphery. This transit is a prerequisite for the secondary or leptotene spermatocyte to enter meiotic division producing haploid round spermatids which undergo a series of differentiation steps to ultimately generate spermatozoa

TGF $\beta$ ligand signalling is a crucial regulator of spermatogenesis. Among these factors, Activin is known to play a role at least in early stages of postnatal testicular development in mice. Evidence supports a role of Activin in determining Sertoli cell numbers and spermatogonial maturation. ${ }^{22,23}$ Activin expression in the testis peaks in postnatal week 1 and is diminished after the establishment of the full complement of SC numbers by the end of week 2. ${ }^{24} \mathrm{~A}$ measurement of total Activin protein per testis however demonstrates a trough around day 20 and then increased expression from day 30 onwards. Moreover, in-situ hybridization data localises $\operatorname{Inh} B A$ and $\operatorname{Inh} B B$ transcripts in SC, spermatogonium as well as spermatocytes in the adult. ${ }^{24}$ There is also evidence that Activin is produced in peritubular myoid cells. $^{23}$ These findings support a role for Activin in testicular function beyond SC number regulation in the early postnatal testis. Indeed, mice with a deletion of ActRIIA show a significant impairment of testicular growth and spermatogenesis underscoring the importance of TGF $\beta$ ligands that bind to this receptor in testicular development and function. ${ }^{8,25}$

Since the sActRIIB ligand-trap is known to bind Activin, ${ }^{26}$ and that Activin action is crucial for testicular development and function we sought to investigate whether increase in muscle mass induced by sActRIIB is accompanied by changes in testicular biology. We have investigated whether there is a developmental window (age of mice) within which these effects are restricted and whether they are reversible over periods of nonintervention. Finally, to compare and contrast the testicular effect of inhibition of Activin or Myostatin we 
Table 1. Antibody details

\begin{tabular}{|c|c|c|c|}
\hline $\begin{array}{l}\text { Primary antibodies } \\
\text { Antigen }\end{array}$ & Species & Dilution & Supplier \\
\hline PCNA & Mouse & $1: 200$ & Cell signalling Technology \#25865 \\
\hline PLZF (D-9) & Mouse & $1: 250$ & Santa Cruz Biotechnology \#sc-28319 \\
\hline Stra8 & Rabbit & $1: 1000$ & Abcam ab49405 \\
\hline Sox9 & Rabbit & $1: 1000$ & Abcam ab185966 \\
\hline AQP3 & Rabbit & $1: 800$ & Abcam ab125219 \\
\hline $\begin{array}{l}\text { Phospho-Smad2 } \\
\text { (Ser465/467)/Smad3 } \\
\text { (Ser423/425) }\end{array}$ & Rabbit & $1: 1000$ & Cell signalling Technology \#8828 \\
\hline Smad2/Smad3 & Rabbit & $1: 1000$ & Cell signalling Technology \#3102 \\
\hline Phospho-AKT (Ser473) & Rabbit & $1: 1000$ & Cell signalling Technology \#4060 \\
\hline AKT & Rabbit & $1: 1000$ & Cell signalling Technology \#9272 \\
\hline $\begin{array}{l}\text { Phospho-p44/42 MAPK } \\
\text { (Erk1/2) (Thr202/Tyr204) }\end{array}$ & Rabbit & $1: 1000$ & Cell signalling Technology \#9101 \\
\hline p44/42 MAPK (Erk1/2) & Rabbit & $1: 1000$ & Cell signalling Technology \#4695 \\
\hline Alpha-tubulin & Mouse & $1: 1000$ & Cell signalling Technology \#3873s \\
\hline Secondary antibodies & Species & Dilution & Supplier \\
\hline AlexaFluor 488 anti-mouse & Goat & $1: 200$ & Invitrogen \\
\hline Rabbit anti mouse IgG HRP & Rabbit & $1: 200$ & DAKO \#PO260 \\
\hline Goat anti-rabbit IgG HRP & Goat & $1: 5000$ & ThermoFisher scientific \#65-6120 \\
\hline
\end{tabular}

have also investigated the testicular phenotype of Mstn null mice. We show that sActRIIB treatment decreases testis size at any stage of the life of mice. Furthermore, we show that the testis phenotype persists longer than muscle hypertrophy following the withdrawal of sActRIIB treatment.

\section{Materials and Methods}

\section{Ethical approval}

The experiments were performed under a United Kingdom Home Office project licence, in agreement with the Animals (Scientific Procedures) Act 1986. The University of Reading Animal Care and Ethical Review Committee approved all procedures. Animals were humanely sacrificed via Schedule 1 killing.

\section{Animal maintenance}

All mice used were bred and maintained in accordance to the Animals (Scientific Procedures) Act 1986 (UK) and approved by the Biological Resources Unit of University of Reading. Post-natal Myostatin/Activin block was induced via intraperitoneal (IP) injection with $10 \mathrm{mg} / \mathrm{kg}$ of sActRIIB-Fc (hereafter sActRIIB) twice weekly. The age of initial injection varied among different studies. CD1 mice were obtained from Jackson laboratories and bred for pups. $10 \mathrm{mg} / \mathrm{kg}$ sActRIIB-Fc was injected at P17 until P37 in all CD1 mice studies with three collection points; P37, P56 and P180. $\mathrm{Mstn}^{+/-}$and $\mathrm{Mstn}^{-/}$tissues were obtained from Versailles Saint-Quentin-enYvelines University at 7.5 months of age. ERCC1 $\Delta /$ mice were bred as previously described (27)with
sActRIIB injections given from 7 weeks of age until 16 weeks.

\section{Histological analysis and immunohistochemistry}

Following dissection, testes were weighed and fixed in $4 \%$ PFA prior to $4^{\circ} \mathrm{C}$ storage. PFA fixed testis were embedded in paraffin following a series of ethanol and histoclear stages. $8 \mu \mathrm{m}$ sections were cut using a Leica RM2155 rotary microtome prior to dewaxing in Xylene. Rehydration with ethanol stages was then performed prior to antigen retrieval in $0.01 \mathrm{M}$ citrate buffer pH6. Blocking buffer (10\% FBS in TBS-Tx) was incubated on sections for 45 minutes at room temperature prior to incubation with primary antibody overnight at $4^{\circ} \mathrm{C}$. Sections were subsequently washed in PBS-Tx three times prior to incubation with secondary antibody. Secondary antibodies attached to a fluorophore were used for detection of all antibodies except PLZF and PCNA which were detected using the Vectastain ABCHRP kit with a Vector NOVARed HRP substrate. All primary antibodies being detected were subsequently incubated with a secondary antibody for 1 hour at room temperature prior to detection. Details of primary and secondary antibodies are given in Table 1 .

\section{Haematoxylin and eosin}

Teste sections were dewaxed in xylene prior to rehydration in ethanol stages. Harris heamatoxylin solution (Sigma HHS16) was then incubated on the slides for 2 minutes prior to acidic alcohol and tap water for blueing. Eosin solution (Sigma-Aldrich 318906) was 
incubated on sections for 2 minutes prior to dehydration in ethanol and clearing with xylene. Slides were mounted with a coverslip and DPX mounting media.

\section{TM4 Sertoli cell line analysis}

In 24 well plate dishes approximately 70,000 TM4 cells were seeded per well. Cells were allowed to attach to the bottom of the dish for approximately 12 hours in cultured media (DMEM/F12-Gibco 11330032- supplemented with $10 \%$ FBS and 100Units/mL Penicillin $100 \mathrm{ug} / \mathrm{mL}$ Streptomycin). After the 12 hours, culture media was supplemented with either 10ng/mL Activin A, 10ng/mL GDF8 or $10 \mathrm{ng} / \mathrm{mL}$ GDF11. After 1 hour of supplementation, cell nuclei were stained with Nuc-Blue Life cell dye (Invitrogen) and images acquired on a Leica inverted microscope with UV filter. After 24 hour of culture cells were fixed with $4 \%$ PFA pH7.4 stained with DAPI and imaged on a Leica upright microscope. Cells were counted using the particle analysis function incorporated on public domain image analyses software Fiji. TM4 cells similarly treated for $15 \mathrm{~min}$ were collected to prepare protein lysates as described below.

\section{Testis Protein Lysate Preparation and Western Blotting}

Frozen tissues were homogenised in detergent-free lysis buffer (50mM Tris $\mathrm{pH} 7.5,150 \mathrm{mM} \mathrm{NaCl}, 1 \mathrm{mM}$ $\mathrm{Na} 3 \mathrm{VO} 4,1 \mathrm{mM} \mathrm{NaF}, 1 \mathrm{mM} \mathrm{MgCl} 2$ ) using a tissue grinder. These tissue lysates were then adjusted to $0.5 \%$ Triton X-100, 0.5\% NP40 and incubated on ice for 15 minutes with intermittent mixing. TM4 cells were collected and directly lysed in lysis buffer as above but also containing $0.5 \%$ TritonX-100 and $0.5 \%$ NP40 and incubated on ice for $15 \mathrm{~min}$ with intermittent mixing. Lysates were clarified by centrifugation at $500 \mathrm{~g}$ and protein content estimated by Bradford assay (Bio-Rad, Hercules, CA, USA). Protein western blotting was performed using standard techniques. Briefly, $100 \mu \mathrm{g}$ lysates were separated on $10 \%$ acrylamide gels and then transferred to nitrocellulose membranes (Amersham Biosciences, Piscataway, NJ, USA). Membranes were washed in TBST $(50 \mathrm{mM}$ Tris $\mathrm{pH} 7.5,150 \mathrm{mM} \mathrm{NaCl}$, $0.05 \%$ Tween 20) and incubated with blocking buffer (5\% non-fat dry milk in TBST) at room temperature for 1 hour. Protein blots were incubated with primary antibodies, used at 1:1000 in blocking buffer, overnight at $4 \mathrm{oC}$. Membranes were then washed three times with TBST and incubated with appropriate horseradish peroxidise conjugated secondary antibodies (Sigma, St. Louis, MO, USA). The membranes were again washed four times and the immunoblot was visualized by ECL (Amersham Biosciences, Piscataway, NJ, USA). Immunoreactive protein bands were analysed using the public domain Image $\mathbf{J}$ program. Blots were stripped and reprobed with additional antibodies using standard methods. Briefly, stripping buffer $(62.5 \mathrm{mM}$ Tris $\mathrm{pH} 6.8$, $2 \%$ SDS, $100 \mathrm{mM} \beta$-mercaptoethanol) was pre-heated to $50 \mathrm{OC}$ in a water bath and membranes were placed into containers containing $100 \mathrm{ml}$ stripping buffer and left to incubate at $50 \mathrm{oC}$ for 40 minutes with gentle shaking. After membranes were removed from stripping buffer, they were washed extensively in TBST. Membranes were subsequently blocked again in 5\% milk block for 1 hour before additional primary antibodies were applied.

\section{Statistical analysis}

Data are presented as mean +/- SE. Significant differences between two groups were performed by the Student's t-test for independent variables. Normal distribution of data was first determined. Thereafter oneway analysis of variance followed by Bonferroni's multiple comparison tests was used for statistical differences between groups of more than two. Statistical analysis was performed on GraphPad Prism 5 with statistically significant differences considered at $\mathrm{P}<0.05$.

\section{Results}

Acute impact of attenuation of Activin Receptor IIB signalling on the development of skeletal muscle and the testis

Inhibiting ligand binding to the Activin Receptor IIB has been demonstrated in a number of differing contexts to induce muscle growth in adult mice. ${ }^{27-29}$ Here we examined the outcome of exposing very young mice. This is a period of development akin to the proposed optimum window for treating boys with DMD, to the soluble form of the Activin type IIB receptor (sActRIIB), which has been previously shown to prevent both Activin and Myostatin from binding to their receptor. Mice were injected twice weekly through the intraperitoneal route from p17 to p35 and culled at p37 (Fig. 1A). The body weights were not significantly changed by sActRIIB treatment (Fig. 1B). In contrast all the muscles examined had increased in weight, exemplified by the $20 \%$ increase in gastrocnemius mass (Fig. 1C). During the course of tissue recovery, we noticed that the testis were smaller in the sActRIIB treated group compared to those receiving vehicle (PBS) (Fig. 1D). The testis from the sActRIIB cohort were also smaller when their weight was expressed as a ratio to body weight (data not shown). We investigated the underlying cellular cause for the decreased testis weight through histological and immunocytochemical investigations. Individual seminiferous tubules were readily identifiable in both cohorts with expected peritubular, basal, adluminal cellular distribution and lumen. The tubule diameters from sActRIIB treated mice, however, were significantly smaller after sActRIIB treatment compared to controls (Fig. 1, E and H). Lumen area was smaller in sActRIIB treated samples than those of controls but did not reach statistical significance (Fig. 1F). Finally the area of the cellular component of each tubule was measured and revealed that this parameter was lower in sActRIIB treated samples compared to controls (Fig. 1G). These findings suggest a reduction of spermatogenesis upon sActRIIB treatment. We then sought to elucidate whether sActRIIB treatment led to alterations in cellular 

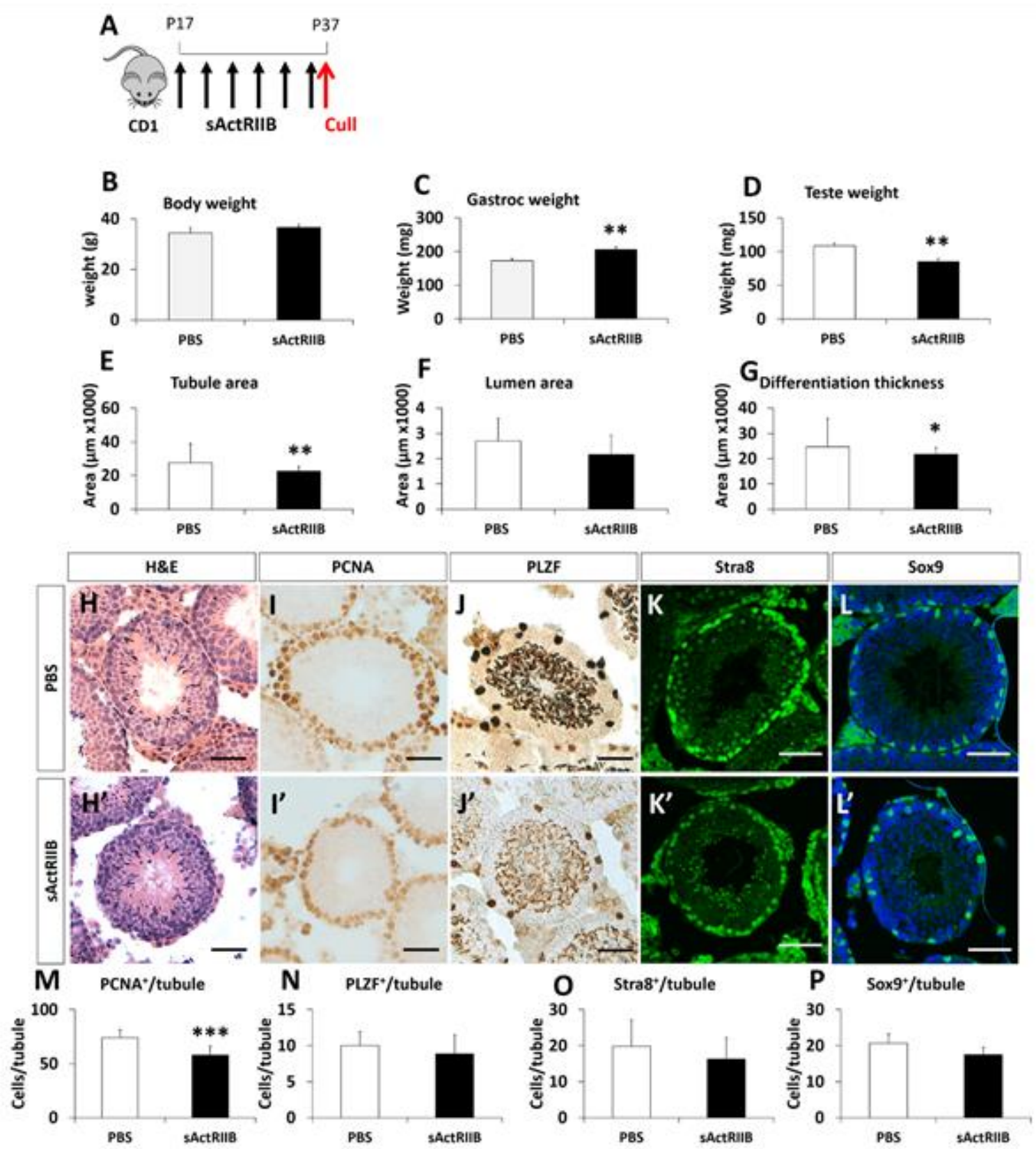

Fig 1. SActRIIB causes a reduction in testicular development in young CD1 mice. (A) Schematic of experimental design. Black arrows injection of $10 \mathrm{mg} / \mathrm{kg}$ sActRIIB. Red arrows indicate time of cull. (B) Body weight at P37. (C) Gastrocnemius mass. (D) Teste mass. (E) Average tubule area. $(F)$ Average lumen area. $(G)$ Average differentiation thickness. $(H) H \& E$ of seminiferous tubule in PBS \& sActRIIB treated showing smaller tubule with SActRIIB. (I) PCNA positive cells in PBS \& sActRIIB tubules showing a reduction in sActRIIB tubule. (J) PLZF positive cells in $P B S \&$ \& sActRIIB tubules. (K) Stra8 positive cells in PBS \& sActRIIB tubules. $(L)$ Sox9 positive cells in PBS \& sActRIIB tubules. (M) Quantification of PCNA positive cells per tubule. (N) Quantification of PLZF positive cells per tubule. $(O)$ Quantification of Stra8 positive cells per tubule. (P) Quantification of Sox9 positive cells per tubule. $n=5$ PBS treated male mice, $n=6$ sActRIIB treated male mice. Scale for all images represents $50 \mu \mathrm{m}$. Student's $t$-Test used for statistical significance. ${ }^{*} p<0.05, * *<0.01$, $* * *<0.001$. H\&E, haematoxylin \& eosin; PCNA, proliferating cell nuclear antigen; PLZF, promyelocytic leukemia zinc finger; Stra 8 , Stimulated By Retinoic Acid 8; Sox9, SRY-Box 9.

distribution or processes in the testis that might cause a likely reduction in spermatogenesis.

sActRIIB treatment decreases proliferation of cells in the testis of young mice

To assess whether the reduction of seminiferous tubule cross section is caused by reduced cell proliferation we quantified the number of proliferating cell nuclear antigen (PCNA) expressing cells. While the location of proliferating cells, adjacent to the basement membrane did not differ, there were significantly fewer proliferating cells in the tubules of sActRIIB treated samples than controls (Fig. 1, I and M). Since proliferating cells, A and B type spermatogonial cells, within tubules originate 


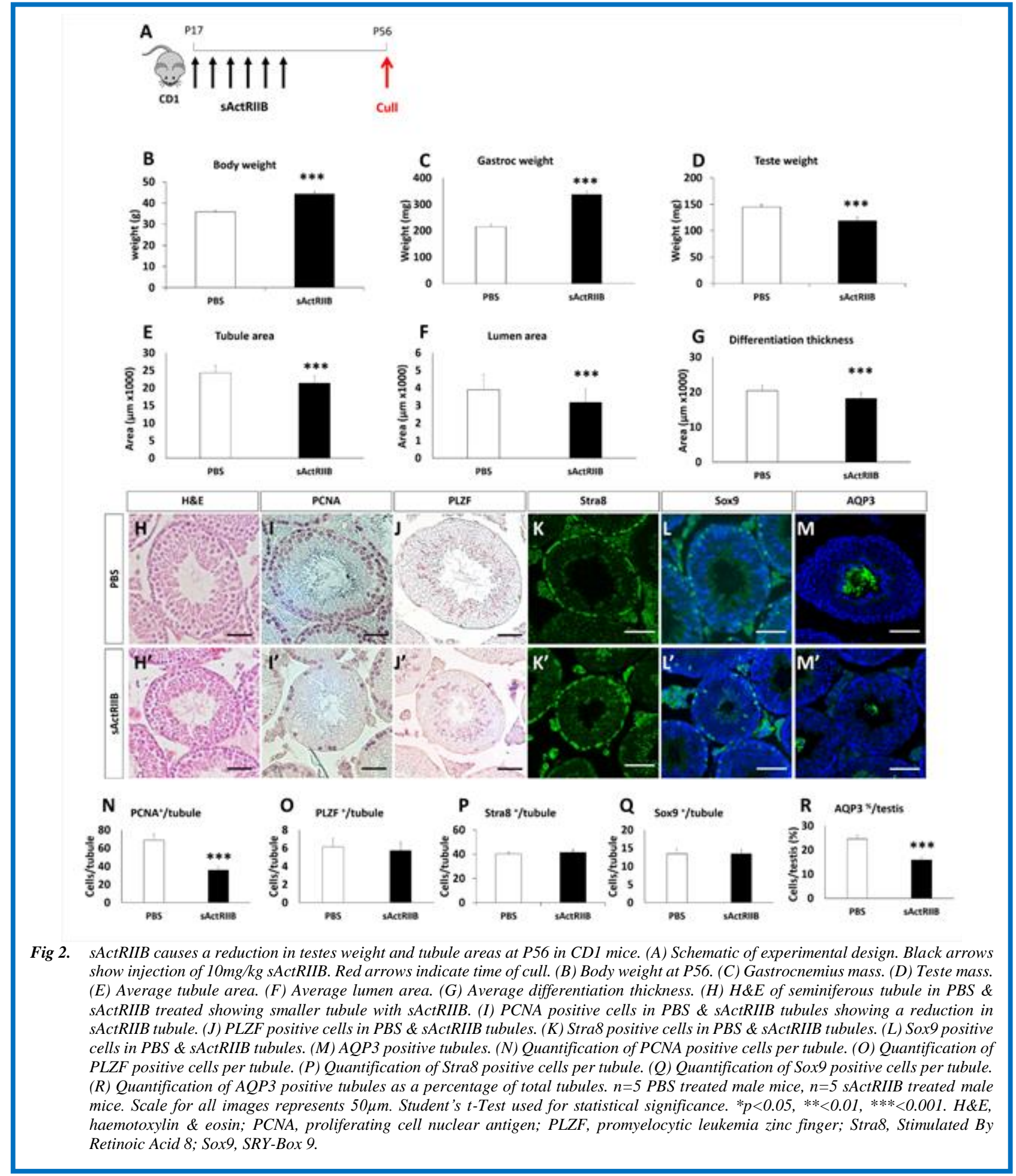

from spermatogonial stem cells (SSC) we quantified the SSC population using PLZF immunostaining, ${ }^{30}$ and found that sActRIIB treatment did not affect spermatogonial stem cell number per tubule (Fig. 1, J and $\mathrm{N}$ ) or the ability of preleptotene spermatocyte to enter meiosis as demonstrated in Fig. $1, \mathrm{~K}$ and $\mathrm{O}$ by Stra8 staining. ${ }^{31}$ Lastly we quantified the number of Sertoli cells, identified by Sox 9 expression, ${ }^{32}$ which supports and thus ultimately limits the extent of spermatogenesis in each tubule and found no differences in this parameter between the sActRIIB treated group and controls (Fig. 1, $\mathrm{L}$ and $\mathrm{P}$ ). Importantly, at this time point we did not see evidence of sperm tails either through $\mathrm{H}$ - E staining or immunostaining with sperm tail specific markers, ${ }^{33}$ including Aquaporin3 (Fig. 1H and data not shown). Of note, there was no effect on Leydig cell numbers by sActRIIB treatment (data not shown). 


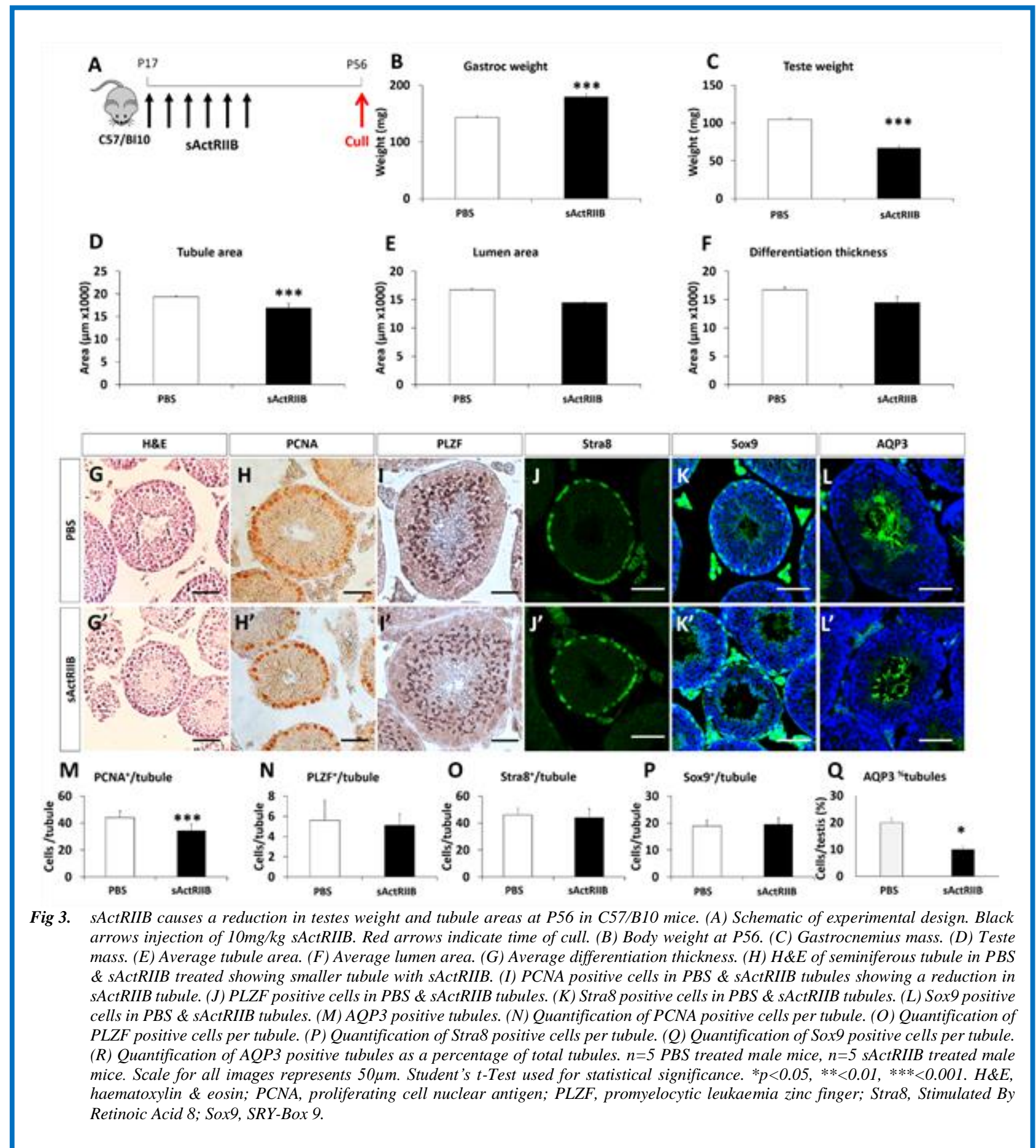

\section{sActRIIB decreases sperm number}

The data from examining the testis of p37 mice demonstrated that sActRIIB, while promoting muscle growth, prevented them from developing to their normal size. While testicular cell proliferation was reduced in mice treated with sActRIIB in the prepubertal stage, we wanted to investigate whether this affects sperm production in the adult. To that end we carried out the same experiment as outlined in Figure 1 except that mice were culled at p56 (adult) since sperm are not fully matured in mice at p37. Examination of body weights of mice treated from $\mathrm{p} 17$ to $\mathrm{p} 35$ and left for another 21 days (to p56) showed that the sActRIIB treated groups were heavier $(23 \%)$ than those injected with PBS (Fig. 2, A and B). Although the gastrocnemius muscle in the sActRIIB group had increased in weight (by 56\%), the testes were lighter (by 19\%) than those of controls (Fig. 2, C and D). Similar to the P37 mice, the cross-section of the seminiferous tubules from treated mice were smaller which was quantitatively verified to show a decrease in 


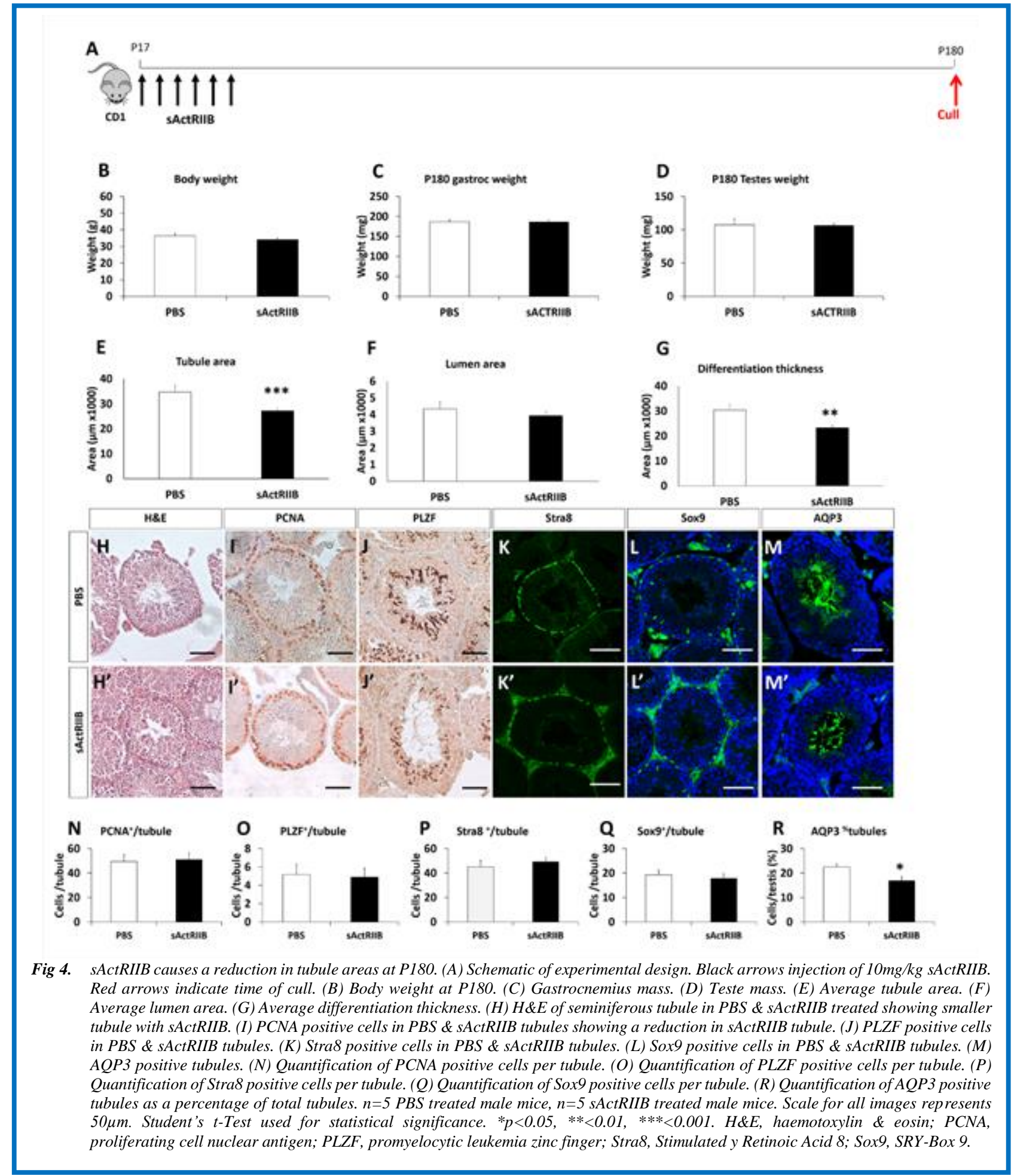

tubule area, lumen area and thickness of spermatogenic area in sActRIIB treated samples (Fig. 2, E-H). Again, like the p37 experiment, the number of proliferating cells was decreased in sActRIIB samples (Fig. 2, I and N) but there was no change in the density of PLZF, Stra8 and Sox9 positive cells (Fig. 2, I-L and O-Q). By p57, however, the control cohort contained matured spermatozoa, as evidenced by tails marked by aquaporin
3 , in the seminiferous tubule lumen which was very significantly reduced in sActRIIB treated samples (Fig. 2, $\mathrm{M}$ and $\mathrm{R}$ ).

We examined whether genetic background was a modifying factor in terms of tissue response to exposure to sActRIIB. We carried out a series of experiments using C57B110 (Fig. 3) as well as the FVB (data not shown) background. In both strains, injection of sActRIIB at p17 
to p35 followed by tissue isolation at p56 (Fig. 3A) resulted in robust muscle mass increase (Fig. $3 \mathrm{~B}$ ) and a decrease in testis weight (Fig. 3C). Again there was a decrease in tubule area, lumen area as well as differentiation area (Fig. 3D-F). As for the CD1 background, immunocytochemical studies revealed a decrease in the number of PCNA and AQP3 containing tubules without any significant changes in PLZF, Stra8 or Sox9 levels (Fig. 3G-Q).

Therefore, treatment of young mice with sActRIIB results in sustained increased skeletal muscle levels but smaller testis which (at least for the CD1 strain) contained less than the normal number of mature spermatozoa.

sActRIIB treatment in young mice results in long term testicular abnormalities

We then investigated whether the reduced spermatogenesis in adult mice caused by a cellular proliferation defect resulting from sActRIIB treatment of pre-pubertal mice, was of a permanent nature or whether this effect was reversible. To that end we injected mice until p35 and examined tissues at p180 (Fig. 4, A-R). Here we failed to detect any significant difference in

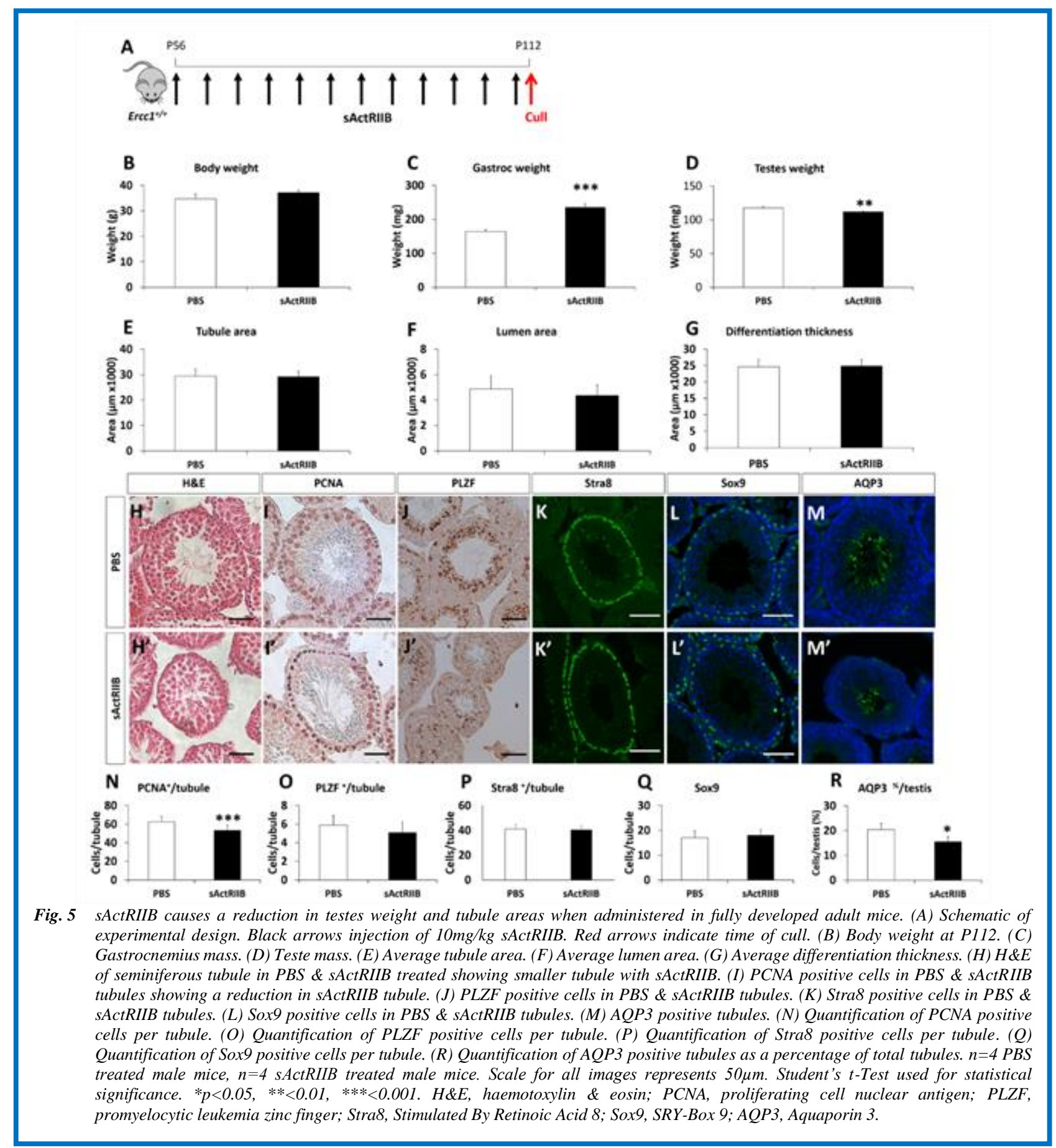


body, muscle or testis weight between the two cohorts. However examination of testis still showed that the sActRIIB cohort displayed lower tubule area and differentiation area compared to the PBS treated mice.
Additionally the sActRIIB cohort contained fewer spermatozoa as quantified through AQP3 staining analyses (Fig. 4, M and R).

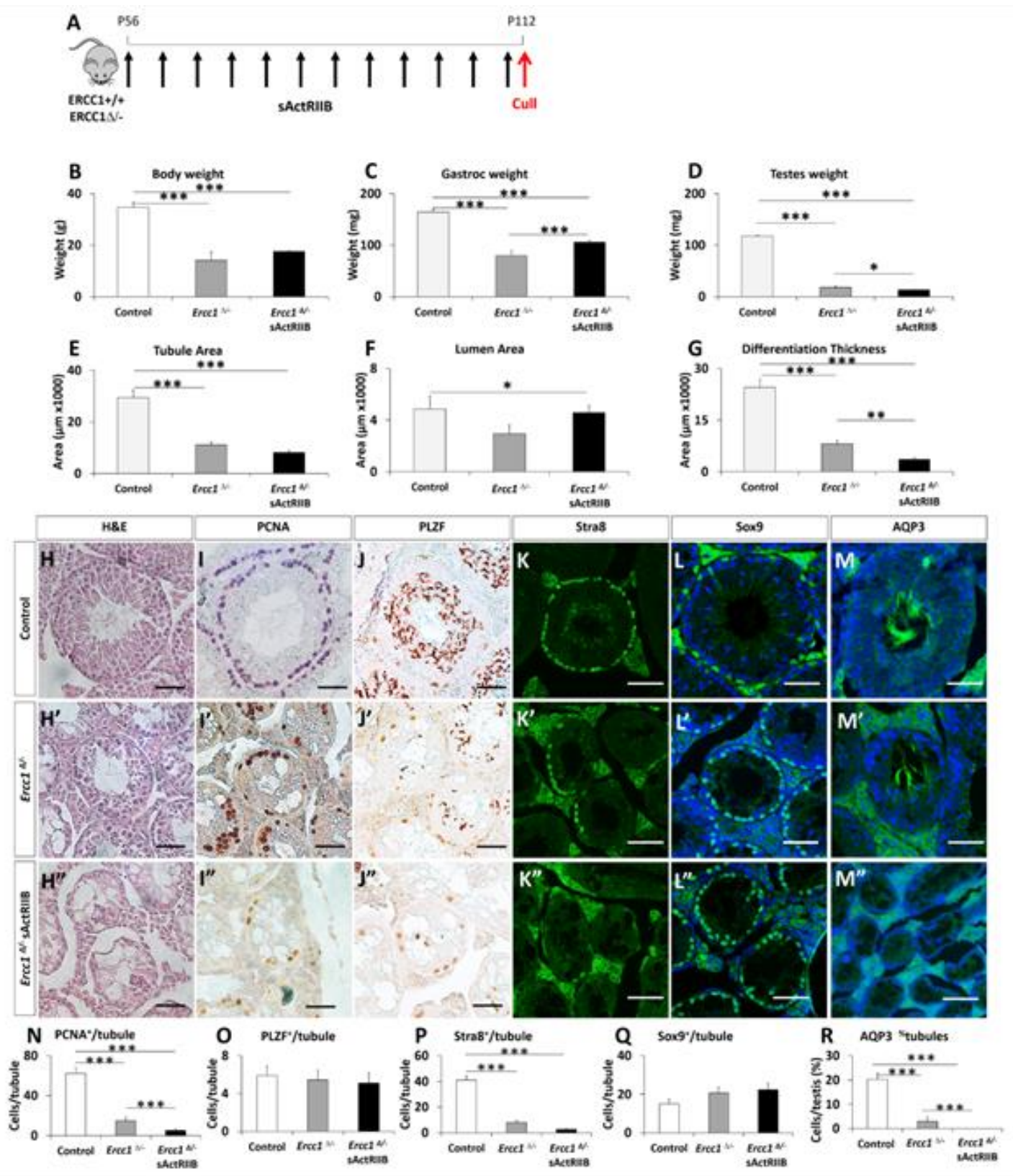

Fig 6. SActRIIB causes a reduction in testes weight and tubule areas in Ercc1L/-treated mice. (A) Schematic of experimental design. Black arrows injection of $10 \mathrm{mg} / \mathrm{kg}$ sActRIIB. Red arrows indicate time of cull. (B) Body weight at P112. (C) Gastrocnemius mass. (D) Teste mass. (E) Average tubule area. $(F)$ Average lumen area. $(G)$ Average differentiation thickness. $(H) H \& E$ of seminiferous tubule in PBS \& sActRIIB treated showing smaller tubule with SActRIIB. (I) PCNA positive cells in PBS \& SActRIIB tubules showing a reduction in sActRIIB tubule. (J) PLZF positive cells in PBS \& sActRIIB tubules. (K) Stra8 positive cells in PBS \& sActRIIB tubules. (L) Sox 9 positive cells in PBS \& sActRIIB tubules. (M) AQP3 positive tubules. (N) Quantification of PCNA positive cells per tubule. (O) Quantification of $P L Z F$ positive cells per tubule. $(P)$ Quantification of Stra8 positive cells per tubule. $(Q)$ Quantification of Sox 9 positive cells per tubule. (R) Quantification of AQP3 positive tubules as a percentage of total tubules. $n=4$ PBS treated male mice, $n=4$ PBS treated ErcclD/-

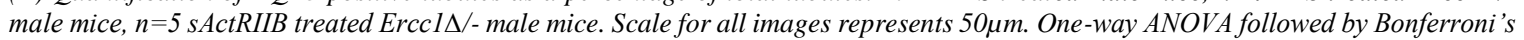
multiple comparison tests, ${ }^{*} p<0.05, * *<0.01$, ***<0.001. H\&E, haematoxylin \& eosin; PCNA, proliferating cell nuclear antigen; PLZF, promyelocytic leukemia zinc finger; Stra8, Stimulated By Retinoic Acid 8; Sox9, SRY-Box 9; AQP3, Aquaporin 3. 
sActRIIB induces testis atrophy in adult mice and in model of aged mice

The experiments conducted thus far were initiated in young mice. We next determined the consequence of sActRIIB treatment on adult mice as well as in a model of extreme ageing. In the first set of these experiments, adult mice (p56) were treated until p110 and thereafter culled at p112 (Fig. 5, A-R). sActRIIB treated mice showed a non-significant increase in body weight but a significant increase in muscle weight compared to controls (Fig. 5, B-C). In contrast, the testis were lighter in the sActRIIB group compared to controls (Fig. 5, D). The sActRIIB group showed non-significant decrease in tubule area, lumen area as well as differentiation area (Fig. 5, E-G). Nevertheless PCNA counts were lower in the sActRIIB group (Fig. 5, N) as were the number of tubules containing AQP3 positive spermatozoa (Fig. 5, $\mathrm{R})$.

Thereafter we examined the consequence of sActRIIB treatment on the $\operatorname{Ercc} \Delta /$ - mice, an established mouse model of ageing. ${ }^{34}$ Mice were injected from p56 (before
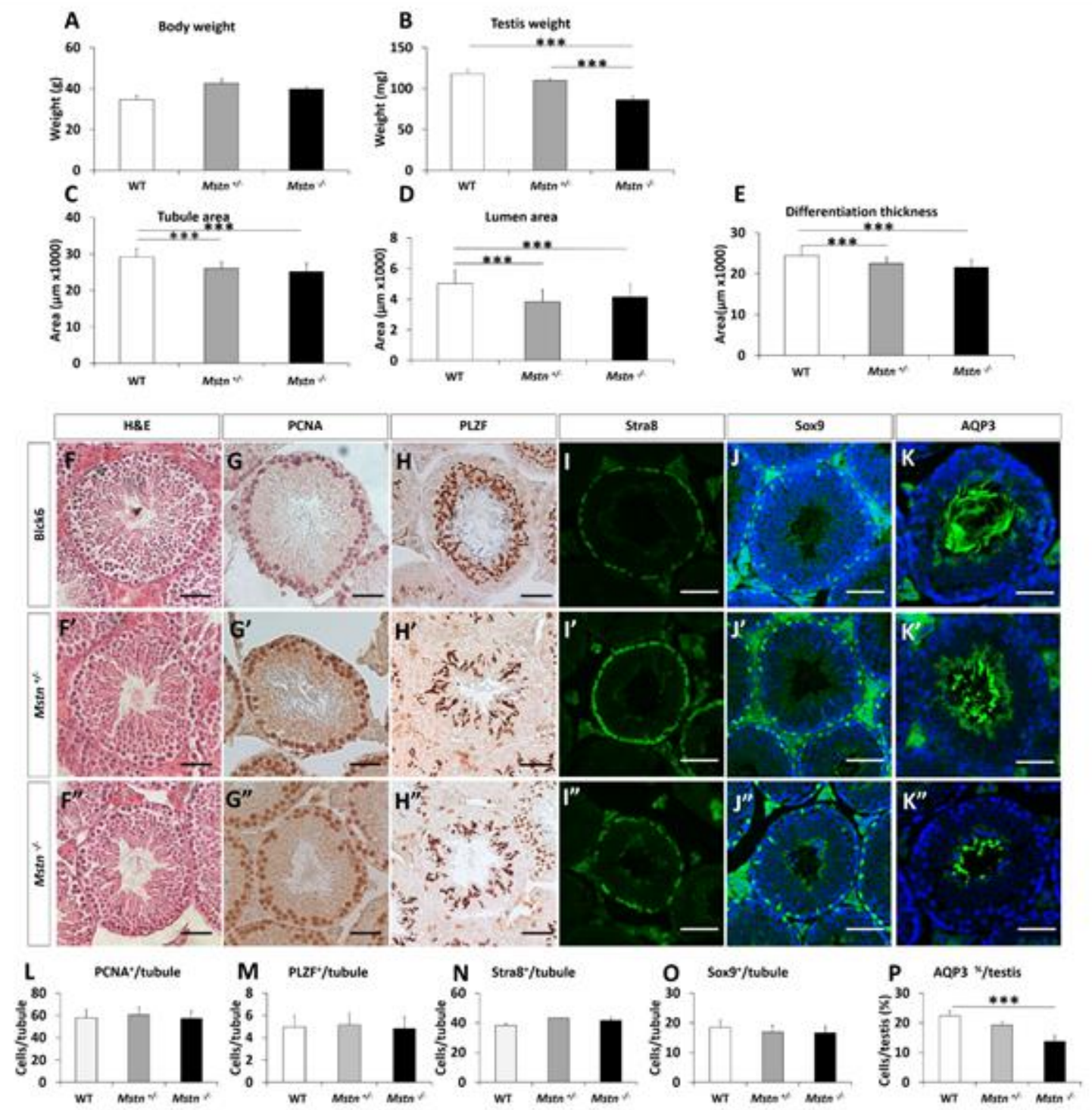

Fig. 7. Testes weight and tubule areas are reduced in Mstn heterozygous and Mstn null mice. (A) Body weight at 7.5 months. (B) Teste mass. (C) Average tubule area. (D) Average lumen area. (E) Average differentiation thickness. $(F) H \& E$ of seminiferous tubule in PBS \& sActRIIB treated showing smaller tubule with sActRIIB. $(G)$ PCNA positive cells in PBS \& sActRIIB tubules showing a reduction in sActRIIB tubule. (HJ) PLZF positive cells in PBS \& sActRIIB tubules. (I) Stra8 positive cells in PBS \& sActRIIB tubules. (J) Sox 9 positive cells in PBS \& sActRIIB tubules. (K) AQP3 positive tubules. (L) Quantification of PCNA positive cells per tubule. (M) Quantification of $P L Z F$ positive cells per tubule. (N) Quantification of Stra8 positive cells per tubule. (O) Quantification of Sox 9 positive cells per tubule. (P) Quantification of AOP3 positive tubules as a percentage of total tubules. $n=4$ PBS treated male mice, $n=5$ Mstn+/- male mice, $n=4$ Mstn-/- male mice. Scale for all images represents 50 $\mu \mathrm{m}$. One-way ANOVA followed by Bonferroni's multiple comparison tests, * $p<0.05$, $* *<0.01$, ***<0.001. H\&E, haematoxylin \& eosin; PCNA, proliferating cell nuclear antigen; PLZF, promyelocytic leukemia zinc finger; Stra8, Stimulated By Retinoic Acid 8; Sox9, SRY-Box 9 
signs of overt ageing) and tissues collected at p112 a time point which we have previous shown to be equivalent of over 2.5 mouse years (Fig. 6A). ${ }^{27}$ Progeric mice displayed lower body weights compared to controls at p112 (Fig. 6B). sActRIIB treatment of progeric mice resulted in a non-significant increase in body weight (Fig. 6B) accompanied by an increase in muscle mass albeit not reaching control levels (Fig. 6C). In contrast, testis weights were lowered in $\operatorname{Ercc} \Delta /$ - mice by sActRIIB (Fig. 6D). Tubule area, lumen area as well as differentiation thickness was smaller in $\operatorname{Ercc} \Delta /$ - testis compared to controls which reduced further following the treatment of Ercc $\Delta /$ - mice with sActRIIB (Fig. 6, E-G). Immunocytochemical analysis revealed crucial interplay between progeric mechanisms and inhibition of TGF $\beta$ signalling in the $\operatorname{Ercc} \Delta /$ - mice. Whereas levels of proliferating cells, number of cells undergoing meiosis and mature spermatozoa were lower in the $\operatorname{Ercc} \Delta /$ - tissue compared to controls, there was no difference between these cohorts in terms of the number of primary germ cells or Sertoli cells (Fig. 6, H-R). Treatment of Ercc $\Delta /$ mice with sActRIIB resulted in a significant decrease in PCNA and AQP3 containing tubules and a nonsignificant reduction in Sertoli cell number. Most interestingly, sActRIIB treatment lead to almost a complete downregulation of AQP3 in $\operatorname{Ercc} \Delta /$ - testes resulting in a total elimination of the sperm production.

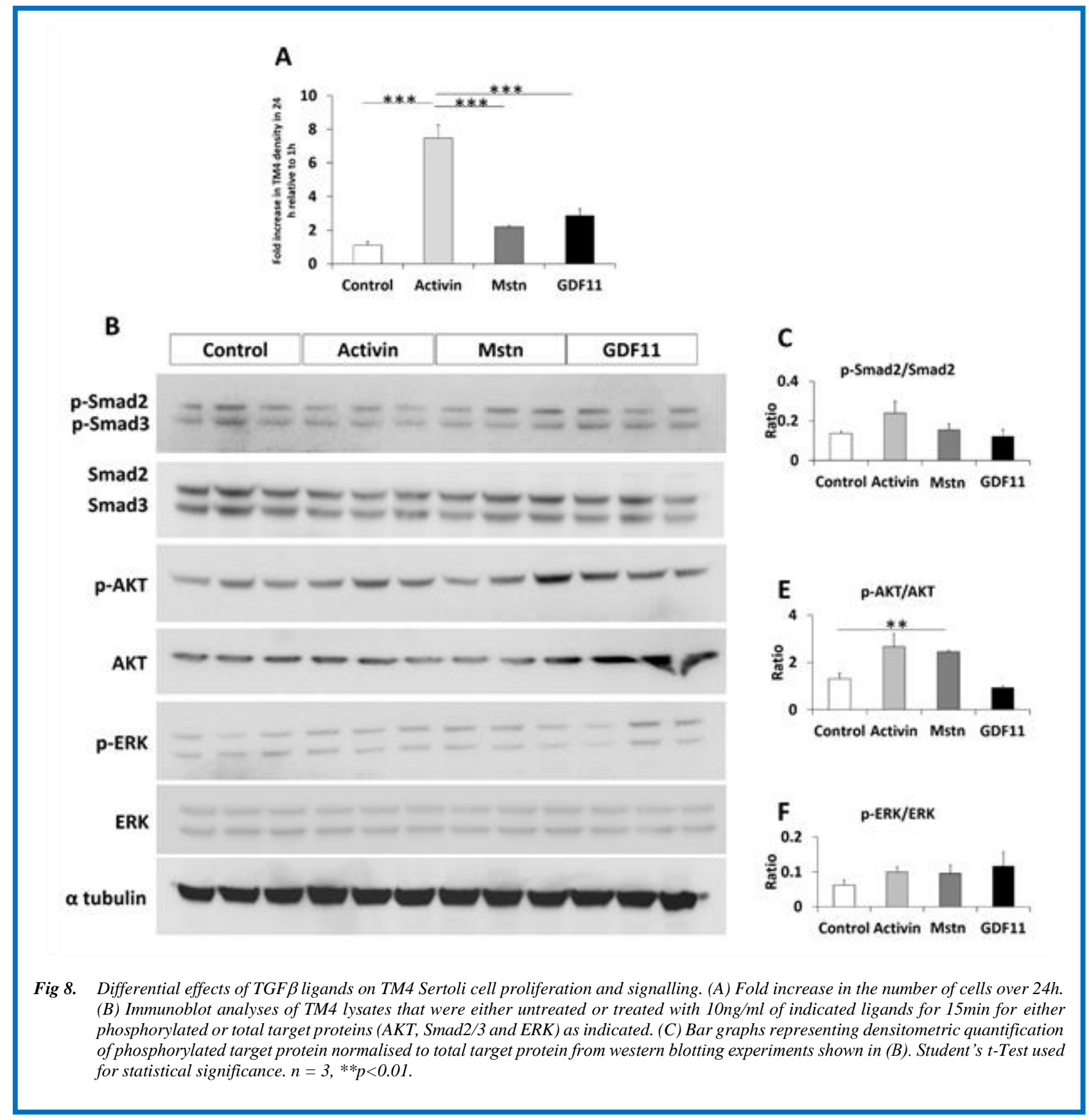


These experiments show that when prepubertal mice are treated with sActRIIB, there is a strong reduction of cellular proliferation which leads to reduced spermatogenesis later in life. This treatment however does not affect the resident spermatogonial stem cell population or their ability to enter meiosis. Further, this effect is not permanent and there is a modest recovery leading to sperm production later in life. Moreover, sActRIIB treatment at any stage in the life of a mouse is likely to decreases testis size and spermatogenesis. Crucially we have also identified an interaction between TGF $\beta$ signalling and ageing of the testis in that sActRIIB treatment synergises with progeroid signalling to prevent germ cell entry into meiosis

\section{sActRIIB induces testicular side-effects in Myostatin null mice}

Our work shows that attenuation of signalling through the ActRIIB can lead to decreased testis mass. One of the molecules that promotes muscle mass through this receptor is Myostatin. To our knowledge a testicular phenotype has not been reported in Myostatin null mice but as a consequence of the results of this study, we examined both the heterozygous and null Myostatin variants. We found that both the heterozygous and homozygous Myostatin mutants had lower testis weights compared to WT litter mates (Fig. 7, A-B). The testis from the nulls were lighter than those from heterozygous mice but failed to reach statistical significance. Histological examination of the tissue revealed that both the heterozygous and null mice had decreased tubule, lumen and differentiation areas compared to WT litter mates (Fig. 7, C-F). Immunocytochemical studies revealed a decrease in the number of AQP3 containing tubules without any significant changes in PCNA, PLZF, Stra8 or Sox9 levels (Fig. 7, G-P).

Postnatal Sertoli cell numbers are regulated by FSH, IGF, testosterone, and Activin action. Since sActRIIB can inhibit Activin, Myostatin and GDF11 action, we addressed whether these ligands affect cellular proliferation in a Sertoli cell line, TM4. As shown in Fig. 8, A, only Activin induces TM4 cellular proliferation and not Mstn or GDF11. Importantly, when investigating proximal signalling effects of these three ligands on TM4 we found only Activin to significantly induce both Smad2 and AKT activation (Fig. 8, B and C). The lack of any effect of sActRIIB treatment on Sertoli cell numbers in vivo might be due to the fact that the injection regime started at p17, and Sertoli cell proliferation is known to occur up to $\mathrm{p} 15$ in mice. ${ }^{36}$ Since Myostatin does not induce Smad2 phosphorylation in TM4 cells (Fig. 8, B and $\mathrm{C}$ ), yet show a similar phenotype as that of mice treated with sActRIIB and since sActRIIB might not traverse the blood testis barrier, it is likely that the constellation of testicular defects described in this study might be due to the reduced effect of Activin and Myostatin action on cells of the spermatogonial lineage. Crucially, however Activin inhibition appears to affect cells up to the primary spermatocytes, whereas the effect of Myostatin deletion is seen only in later stages.

\section{Discussion}

ACE-031, a molecule similar to the sActRIIB used in this study, was progressed into a phase II clinical trial on boys with DMD. However the study was stopped due to safety concerns related to epitaxis and telangiectasias. ${ }^{37}$ Previous work has reported that a similar molecule to ACE-031 and sActRIIB used in our study induced side effects related to the spleen and pancreas. ${ }^{21}$ In this study we hypothesised that testis development may be affected by sActRIIB given the important role that Activin has on this organ. Our study shows that sActRIIB promotes robust muscle growth in a matter of 20 days in mice. However this was accompanied by a decrease in testis weight. This effect was independent to the genetic background of mice, since here we examined three routinely used strains (CD-1, C57/B110 and FVB). Examination of testicular structure showed that sActRIIB inhibited the proliferation of cells in testis without impacting on the number of germ cells, undergoing meiosis or Sertoli cells. We show that following cessation of sActRIIB treatment, the rate of muscle growth in treated mice is reduced compared to untreated mice such that by 145 days after last treatment both the treated and untreated mice have the same muscle mass (Figure 4). Importantly, although the muscle phenotype induced by sActRIIB treatment has been erased at this time, the testis phenotype has not. These results show that positive outcomes of sActRIIB treatment are shorter lived than that detrimental impact on the testis.

Importantly, we show that both mature wild-type mice an ageing model undergo muscle hypertrophy and testicular atrophy following sActRIIB treatment. The most pronounced impact of the treatment in terms of potential testicular function was in fact in the model of aged mice where sActRIIB totally eliminated the formation of AQP3 positive sperm. We believe that the side effects detected after the application of sActRIIB at any stage of life is highly relevant since therapies based on attenuating Myostatin/Activin function are being developed for juvenile (e.g. DMD) ${ }^{38,39}$ adult (heart failure) ${ }^{39}$, and late onset diseases, e.g. inclusion body myostis, oculopharyngeal muscular dystrophy, ${ }^{40,41}$ as well as sarcopenic obesity. ${ }^{42}$

The data from our study regarding the inhibition of testis development can be explained by on-target effects that should be expected for a molecule that inhibits Activin. ${ }^{45}$ It is surprising that, to the best of our knowledge, a testis phenotype has not been described in other studies using similar molecules which bring about muscle growth. In many studies there is no mention that the testis were examined and even when they were, there is not data presented regarding their mass. ${ }^{46-47}$ It is also not clear whether testicular size was examined in boys on the ACE-083 trial. However, we suggest that in future this metric be included in any clinical trial that relies on 
Myostatin/Activin neutralisation as the mode of action to promote muscle growth.

Although both sActRIIB treatment of wild-type mice and Myostatin deletion shows a reduction of spermatogenesis in mice, the mechanism of pathogenesis is likely to be different. Although Activin is known to play crucial local roles in both the pituitary and the ovary, sActRIIB treatment does not affect the ovarian cycle in mice (not shown) suggesting that the working of the hypothalamuspituitary-gonad (HPG) axis is intact even in the presence of a circulating inhibitor of Activin. Given this, therefore, it is not expected that sActRIIB treatment affects pituitary function in the male. Contrasting the ovary, however, testicular function of spermatogenesis is strongly affected by sActRIIB most likely due to the abrogation of Activin action on the spermatogenic cells themselves. Removal of sActRIIB treatment restores spermatogenesis supporting a partially reversible nature of this brake on the process. This reversibility is expected since sActRIIB treatment does not affect SC or germ cell numbers. Contrasting from the $\mathrm{Msth}^{-/}$mouse testis, however, there is a clear reduction of PCNA positive cells close to the basement membrane inside the seminiferous tubule upon sActRIIB treatment. This is indicative of reduction of spermatogonial proliferation which then suggests that the reduction of testicular size and function in the injected model is due to early inhibition of spermatogenesis. The effectiveness of sActRIIB as a modulator of Activin function, demonstrated by the reduction of Smad2 activation, provides a possible therapeutic strategy to alleviate testicular seminoma development. It has been shown that when gonocyte differentiation to spermatogonia is compromised, the gonocytes produce cancer in-situ or CIS cells within the testis. ${ }^{45}$ These cells develop into seminoma or non-seminoma origin cancers. Importantly, at every stage of the differentiation of the gonocyte to testicular cancer Smad2/3 activation appears to be a common factor. This activation might be effectively contained or inhibited by SActRIIB treatment.

In addition to effects on the testis, it is likely that the ligand trap might affect Activin action elsewhere in the body in a context-dependent manner. While angiogenesis, liver and kidney function might also be affected, a pronounced behavioural effect is also expected upon sActRIIB injection. There is clear evidence of ActRIIB expression in the amygdala indicating that Activin might play a role in anxiety-type behaviour. Moreover, it is known that the presence of Activin has a calming effect on the afferents from the amygdala whereas absence or reduction of Activin can lead to aggressive behaviour stemming from heightened anxiety. In support of this, we have found that sActRIIB treated male mice show highly aggressive behaviour (not shown). Our sActRIIB injection protocol, therefore, presents a model system to further investigate behavioural issues such as anxiety and anxiety-related aggression.
Our study details an experimental intervention in mice that leads to the development of muscularity but at the same time induces testicular atrophy. A similar outcome has been described in men who abuse androgenic anabolic steroids. ${ }^{53,54}$ The co-presentation of these two traits in an organism are in conflict when viewed from evolutionary fitness perspective as big muscles in men are deemed indicative of high genetic quality and positive factors in mate selection by women. ${ }^{55}$ This scenario is called the Mossman-Pacey Paradox which can be succinctly summarised as representing a conflict between masculinity and muscularity. ${ }^{56}$ It is important to recognise that muscle mass increase induced through abuse of androgenic anabolic steroid is also associated with a list of other undesirable health issues including acne, gynaecomastia and most importantly mortality. ${ }^{53}$ Future work will investigate these features in our mouse model of muscle growth induced by sActRIIB. Most importantly the work presented here should be taken as a warning sign for those who want to use Myostatin/Activin inhibitors for vanity purposes.

In summary, we demonstrate that attenuation of Myostatin/Activin signalling delivers significant muscle growth at any stage in the life of a rodent. However, sActRIIB injection induced an abnormal testis phenotype at any stage of the rodents life. The functional consequence of the decrease testis size may impact on fertility. Future work will investigate fertility of mice that have been treated with sActRIIB. Furthermore we will investigate in an appropriately timed manner the role this molecule plays on female reproductive organs.

\section{List of acronyms}

ACE-031 - Acceleron 031 protein

ActRIIA - Actvin receptor IIA

ActRIIB - Actvin receptor IIB

ACVR2A - Actvin receptor $2 \mathrm{~A}$

ACVR2B - Actvin receptor 2B

ALK4 - Activin like kinase4

ALK7 - Activin like kinase7

AQP3 - Aquaporin 3

BMPs - Bone morphogenetic protein

BTB - blood-testis barrier

DMD - Duchenne Muscular Dystrophy

ECL - Enhanced chemiluninescence

GASP-1 - Growth and differentiation factor associated serum protein 1

GDF11 - Growth and differentiation factor 11

IGF-1 - Insuline growth factor 1

$\operatorname{Inh} B A$ - Inhibin BA

$\operatorname{Inh} B B$ - Inhibin BB

IP - intraperitoneal

LTBP-3 - Latent TGF binding protein 3

Mstn - Myostatin

Mstn $^{-/}$- Myostatin null mice

PCNA - Proliferating cell nuscler antigen

PLZF - Promyelocytic Leukemia Zinc Finger

sActRIIB - soluble ActRIIB 
SC - Sertoli cells

Smad2 - SMA Mothers Against Decapentaplegic 2

Sox 9 - SRY Box transcription factor 9

Stra8 - Stimulated By Retinoic Acid 8

TGF $\beta$ - Transforming Gorwth Factor beta

\section{Authors contributions}

Conceptualization OR, AM, KP. Methodology OK, AM, TBH, KP. Validation DV, ML, HM, RB, BD, DW, AM, KP. Formal Analysis DV, ML, HM, RB, BD, DW, AM, KP. Investigation DV, OR, RM, ML, HM, CD, AM, AP, HC-H, RB. Writing DV, ML, BD, DW, AM KP. Visualization DV, Ml, BD, DW, AM, KP. Supervision AM, KP.

\section{Acknowledgments}

Authors are grateful for the Editorial Office of European Journal of Translational Myology guidance during the publication of this paper.

\section{Funding}

This work was supported by the Biotechnology and Biological Sciences Research Council (Grants $\mathrm{BB} / \mathrm{J} 016454 / 1$ to $\mathrm{HCH}$ and $\mathrm{KP}$ and BB/M014878/1 to DV). TBH was supported by the DFG (CRC 1192, CRC1140, CRC 992), by the BMBF (01GM1518C), by the European Research Council-ERC (grant 616891) and by the H2020-IMI2 consortium BEAt-DKD (Innovative Medicines Initiative 2 Joint Undertaking under grant agreement No 115974).

\section{Conflict of Interest}

The authors have no conflicts to disclose.

\section{Ethical Publication Statement}

We confirm that we have read the Journal's position on issues involved in ethical publication and affirm that this report is consistent with those guidelines.

\section{Corresponding Author}

Ketan Patel, Hopkins Building, University of Reading, Reading, RG6 6UB - Phone:+44118 3788079

Email: ketan.patel@ reading.ac.uk

\section{E-mails of co-authors}

Danielle Vaughan: D.Vaughan@pgr.reading.ac.uk OlliRitvos: olli.ritvos@gmail.com

Robert Mitchell: robmitchell1@gmail.com

OliverKretz:o.kretz@uke.de

MaciejLalowski: maciej.lalowski@helsinki.fi

Helge Amthor:

helge.amthor@handimedex.onmicrosoft.com

DavidChambers:david.2.chambers@kcl.ac.uk Antonios Matsakas: Antonios.Matsakas@hyms.ac.uk Arja Pasternack: arja.pasternack@helsinki.fi Henry Collins-Hooper:

Henry.Collins-Hooper@uk.lockton.com

Randy Ballesteros: rballesteros@rvc.ac.uk

Tobias B.Huber: t.huber@uke.de
Bernd Denecke: Bernd.Denecke@rwth-aachen.de

Darius Widera:d.widera@reading.ac.uk

AbirMukherjee: amukherjee@rvc.ac.uk

\section{References}

1. 1.Matsakas A, Patel K. Skeletal muscle fibre plasticity in response to selected environmental and physiological stimuli. Histol Histopathol 2009;24:611-29.

2. Waterlow JC, Jackson AA. Nutrition and protein turnover in man. Br Med Bull 1981;37:5-10.

3. Poortmans JR, Carpentier A, Pereira-Lancha LO, Lancha A, Jr. Protein turnover, amino acid requirements and recommendations for athletes and active populations. Braz J Med Biol Res 2012;45:875-90.

4. Sjoqvist F, Garle M, Rane A. Use of doping agents, particularly anabolic steroids, in sports and society. Lancet 2008;371(9627):1872-82.

5. Windisch PA, Papatheofanis FJ, Matuszewski KA. Recombinant human growth hormone for AIDSassociated wasting. Ann Pharmacother 1998;32:437-45.

6. Borst SE, Lowenthal DT. Role of IGF-I in muscular atrophy of aging. Endocrine 1997;7:61-3.

7. McPherron AC, Lawler AM, Lee SJ. Regulation of skeletal muscle mass in mice by a new TGF-beta superfamily member. Nature 1997;387(6628):8390.

8. Aiello D, Patel K, Lasagna E. The myostatin gene: an overview of mechanisms of action and its relevance to livestock animals. Anim Genet 2018;49:505-19.

9. Lee SJ, McPherron AC. Regulation of myostatin activity and muscle growth. Proc Natl Acad Sci U S A 2001;98:9306-11.

10. Hill JJ, Qiu Y, Hewick RM, Wolfman NM. Regulation of myostatin in vivo by growth and differentiation factor-associated serum protein-1: a novel protein with protease inhibitor and follistatin domains. Mol Endocrinol 2003;17:1144-54.

11. Anderson SB, Goldberg AL, Whitman M. Identification of a novel pool of extracellular promyostatin in skeletal muscle. $J$ Biol Chem 2008;283:7027-35.

12. Matsakas A, Foster K, Otto A, et al. Molecular, cellular and physiological investigation of myostatin propeptide-mediated muscle growth in adult mice. Neuromuscul Disord 2009;19:489-99.

13. Olsen OE, Wader KF, Hella $\mathrm{H}$, et al. Activin A inhibits BMP-signaling by binding ACVR2A and ACVR2B. Cell Commun Signal 2015;13:27.

14. Lach-Trifilieff E, Minetti GC, Sheppard K, et al. An antibody blocking activin type II receptors induces strong skeletal muscle hypertrophy and protects from atrophy. Mol Cell Biol 2014;34:606-18.

15. Hoogaars WM, Mouisel E, Pasternack A, et al. Combined effect of AAV-U7-induced dystrophin 
exon skipping and soluble activin Type IIB receptor in mdx mice. Hum Gene Ther 2012;23:1269-79.

16. Lee SJ, Reed LA, Davies MV, et al. Regulation of muscle growth by multiple ligands signaling through activin type II receptors. Proc Natl Acad Sci U S A 2005;102:18117-22.

17. Sartori R, Schirwis E, Blaauw B, et al. BMP signaling controls muscle mass. Nat Genet 2013;45:1309-18.

18. Cohen S, Nathan JA, Goldberg AL. Muscle wasting in disease: molecular mechanisms and promising therapies. Nat Rev Drug Discov 2015;14:58-74.

19. Koncarevic A, Kajimura S, Cornwall-Brady M, et al. A novel therapeutic approach to treating obesity through modulation of TGFbeta signaling. Endocrinology 2012;153:3133-46.

20. Liu H, Zhang R, Chen D, et al. Functional redundancy of type II BMP receptor and type IIB activin receptor in BMP2-induced osteoblast differentiation. J Cell Physiol 2012;227:952-63.

21. Latres E, Mastaitis J, Fury W, et al. Activin A more prominently regulates muscle mass in primates than does GDF8. Nat Commun 2017;8:15153.

22. Boitani C, Stefanini M, Fragale A, Morena AR. Activin stimulates Sertoli cell proliferation in a defined period of rat testis development. Endocrinology 1995;136:5438-44.

23. Buzzard JJ, Farnworth PG, De Kretser DM, et al. Proliferative phase sertoli cells display a developmentally regulated response to activin in vitro. Endocrinology 2003;144:474-83.

24. Buzzard JJ, Loveland KL, O'Bryan MK, et al. Changes in circulating and testicular levels of inhibin $\mathrm{A}$ and $\mathrm{B}$ and activin A during postnatal development in the rat. Endocrinology 2004;145:3532-41.

25. Kumar TR, Varani S, Wreford NG, et al. Male reproductive phenotypes in double mutant mice lacking both FSHbeta and activin receptor IIA. Endocrinology 2001;142:3512-8.

26. del Re E, Sidis Y, Fabrizio DA, et al. Reconstitution and analysis of soluble inhibin and activin receptor complexes in a cell-free system. J Biol Chem 2004;279:53126-35.

27. Alyodawi K, Vermeij WP, Omairi S, et al. Compression of morbidity in a progeroid mouse model through the attenuation of myostatin/activin signalling. J Cachexia Sarcopenia Muscle 2019. 10:662-686. doi: 10.1002/jcsm.12404. Epub 2019 Mar 27.

28. Relizani K, Mouisel E, Giannesini B, et al. Blockade of ActRIIB signaling triggers muscle fatigability and metabolic myopathy. Mol Ther. 2014;22:1423-33.

29. Omairi S, Matsakas A, Degens H, et al. Enhanced exercise and regenerative capacity in a mouse model that violates size constraints of oxidative muscle fibres. Elife 2016;5. pii: e16940. doi: 10.7554/eLife. 16940.

30. Costoya JA, Hobbs RM, Barna M, et al. Essential role of Plzf in maintenance of spermatogonial stem cells. Nat Genet 2004;36(6):653-9.

31. Zhou Q, Nie R, Li Y, et al. Expression of stimulated by retinoic acid gene 8 (Stra8) in spermatogenic cells induced by retinoic acid: an in vivo study in vitamin A-sufficient postnatal murine testes. Biol Reprod 2008;79:35-42.

32. Morais da Silva S, Hacker A, Harley V, et al. Sox9 expression during gonadal development implies a conserved role for the gene in testis differentiation in mammals and birds. Nat Genet 1996;14:62-8.

33. Chen Q, Peng H, Lei L, et al. Aquaporin 3 is a sperm water channel essential for postcopulatory sperm osmoadaptation and migration. Cell Res 2011;21:922-33.

34. Vermeij WP, Dolle ME, Reiling E, et al. Restricted diet delays accelerated ageing and genomic stress in DNA-repair-deficient mice. Nature 2016;537(7620):427-31.

35. Oldknow KJ, Seebacher J, Goswami T, et al. Follistatin-like 3 (FSTL3) mediated silencing of transforming growth factor beta (TGFbeta) signaling is essential for testicular aging and regulating testis size. Endocrinology 2013;154:1310-20.

36. Auharek SA, de Franca LR. Postnatal testis development, Sertoli cell proliferation and number of different spermatogonial types in C57BL/6J mice made transiently hypo- and hyperthyroidic during the neonatal period. J Anat 2010;216:57788.

37. Campbell C, McMillan HJ, Mah JK, et al. Myostatin inhibitor ACE-031 treatment of ambulatory boys with Duchenne muscular dystrophy: Results of a randomized, placebocontrolled clinical trial. Muscle Nerve. 2017;55:458-64.

38. Shieh PB. Emerging Strategies in the Treatment of Duchenne Muscular Dystrophy. Neurotherapeutics 2018;15:840-8.

39. Roh JD, Hobson R, Chaudhari V, et al. Activin type II receptor signaling in cardiac aging and heart failure. Sci Transl Med 2019;11(482).

40. Ioannis M, Foivos P, Dimitrios K. A review on the treatment of sporadic inclusion body myositis with Bimagrumab and Alemtuzumab. Int $\mathbf{J}$ Neurosci 2019;129:297-302.

41. Harish P, Malerba A, Lu-Nguyen N, et al. Inhibition of myostatin improves muscle atrophy in oculopharyngeal muscular dystrophy (OPMD). J Cachexia Sarcopenia Muscle 2019;10:1016-26. doi: 10.1002/jcsm.12438. Epub 2019 May 7.

42. Consitt LA, Clark BC. The Vicious Cycle of Myostatin Signaling in Sarcopenic Obesity: Myostatin Role in Skeletal Muscle Growth, Insulin 
Signaling and Implications for Clinical Trials. J Frailty Aging 2018;7:21-7.

43. Tillet E, Bailly S. Emerging roles of BMP9 and BMP10 in hereditary hemorrhagic telangiectasia. Front Genet 2014;5:456.

44. Glasser CE, Gartner MR, Wilson D, et al. Locally acting ACE-083 increases muscle volume in healthy volunteers. Muscle Nerve 2018;57:921-6.

45. Dias V, Meachem S, Rajpert-De Meyts E, et al. Activin receptor subunits in normal and dysfunctional adult human testis. Hum Reprod 2008;23:412-20.

46. Morvan F, Rondeau JM, Zou C, et al. Blockade of activin type II receptors with a dual antiActRIIA/IIB antibody is critical to promote maximal skeletal muscle hypertrophy. Proc Natl Acad Sci U S A 2017;114:12448-53.

47. Rooks D, Praestgaard J, Hariry S, et al. Treatment of Sarcopenia with Bimagrumab: Results from a Phase II, Randomized, Controlled, Proof-ofConcept Study. J Am Geriatr Soc 2017;65:1988-95.

48. Garito $\mathrm{T}$, Roubenoff $\mathrm{R}$, Hompesch $\mathrm{M}$, et al. Bimagrumab improves body composition and insulin sensitivity in insulin-resistant individuals. Diabetes Obes Metab 2018;20:94-102.

49. Rodino-Klapac LR, Haidet AM, et al. Inhibition of myostatin with emphasis on follistatin as a therapy for muscle disease. Muscle Nerve 2009;39:283-96.

50. Johnston H, Baker PJ, Abel M, et al. Regulation of Sertoli cell number and activity by follicle- stimulating hormone and androgen during postnatal development in the mouse. Endocrinology 2004;145:318-29.

51. Mithraprabhu S, Mendis S, Meachem SJ, et al. Activin bioactivity affects germ cell differentiation in the postnatal mouse testis in vivo. Biol Reprod 2010;82:980-90.

52. Istasse L, Van Eenaeme C, Evrard P, et al. Animal performance, plasma hormones and metabolites in Holstein and Belgian Blue growing-fattening bulls. J Anim Sci 1990;68:2666-73.

53. Horwitz H, Andersen JT, Dalhoff KP. Health consequences of androgenic anabolic steroid use. J Intern Med 2019;285:333-40.

54. Knuth UA, Maniera H, Nieschlag E. Anabolic steroids and semen parameters in bodybuilders. Fertil Steril 1989;52:1041-7.

55. Sell A, Lukazsweski AW, Townsley M. Cues of upper body strength account for most of the variance in men's bodily attractiveness. Proc Biol Sci 2017;284(1869).

56. Mossman JA, Pacey AA. The fertility fitness paradox of anabolic-androgenic steroid abuse in men. J Intern Med 2019;286:231-2. doi: 10.1111/joim.12884. Epub 2019 Apr 10.

Submission: December, 05, 2019

Acceptance: December, 05, 2019 